\title{
AC 2008-682: AN ASSESSMENT OF PROBLEM SOLVING PROCESSES IN UNDERGRADUATE STATICS
}

\section{Roman Taraban, Texas Tech University}

Roman Taraban is Professor and Associate Chair in the Department of Psychology at Texas Tech University, Assessment Coordinator for the Texas Tech University Howard Hughes Medical Institute (TTU/HHMI) Biological Sciences Education Program, Member of the Texas Tech Teaching Academy Executive Council, past President of the Society for Computers in Psychology (SCiP), and Associate Editor for the Journal of Educational Psychology. He received his Ph.D. in cognitive psychology from Carnegie Mellon University. His interests are in how undergraduate students learn, and especially, how they draw meaningful connections in traditional college content materials (e.g., textbooks, lectures, multi-media). Address: Department of Psychology, Mail Stop 2051, Texas Tech University, Lubbock, TX, 79409; telephone: 806-742-3711 ext. 247; fax: 806-742-0818; email: roman.taraban@ttu.edu.

\section{Edward E. Anderson, Texas Tech University}

Edward E. Anderson is Professor in the Department of Mechanical Engineering at Texas Tech University where he currently serves as the Ray Butler Distinguished Educator. Since returning to the faculty after several different administrative assignments, including Departmental Chairman, Assistant Dean, and Director of the TTU Teaching, Learning and Technology Center, he has focused upon engineering student learning research with an eye upon how to use these findings to improve traditional and computer-based learning.

\section{Curtis Craig, Texas Tech University}

Curtis Craig is a graduate student in experimental psychology at Texas Tech University, with a disciplinary emphasis on applied cognition.

\section{Jacob Fleming, Texas Tech University}

Jacob Fleming is a graduate student in the experimental psychology program at Texas Tech University.

\author{
Alli DeFinis, Texas Tech University \\ Alli DeFinis is a graduate student in the counseling psychology program at Texas Tech \\ University.
}

\section{Ashlee G. Brown, Texas Tech University}

Ashlee Brown is a graduate student in counseling psychology at Texas Tech University, with an interest in philosophy. 


\title{
An Assessment of Problem Solving Processes in Undergraduate Statics
}

\begin{abstract}
Four well-articulated models that offer structured approaches to problem solving were identified in the engineering research literature. These models provided a conceptual base for the study reported here. Four undergraduates enrolled in statics and two engineering faculty members provided think-aloud data as they solved two statics problems. The data were used to develop a coding system for characterizing engineering students' behavioral and cognitive processes. These codes were used to analyze students' problem solving procedures in a detailed manner, particularly differences between goodand not-so-good problem solvers. The analyses provide a picture of how students and faculty solve problems at a cognitive level, and indicate that published problem-solving models are incomplete in describing actual problem-solving processes.
\end{abstract}

Wankat and Oreovicz" asserted that "engineering education focuses heavily on problem solving." This assertion would find significant agreement among engineering educators. The high proportion of time spent solving textbook problems outside of class by engineering undergraduates has been documented in the engineering research literature ${ }^{2}$. The central place of problem solving in engineering has led some scholars to inquire about the nature of effective problem solving, asking about the processes that underlie good problem solving procedures. Engineering educators have also developed didactic models meant to guide classroom practices. The research presented here is based on four well-articulated models that offer structured approaches to problem solving. The models have been developed as a response to students' use of a "hodgepodge of tricks" to solve statics, dynamics, and thermodynamics problems, and they were regarded by their authors as useful to students in developing good problem solving skills. Therefore the models were considered appropriate for an empirical study of problem solving by undergraduates.

The goals of this study were to develop a descriptive language for characterizing engineering students' behavioral and cognitive processes related to problem solving. This descriptive language was developed as a coding system that was used to analyze students' problem solving procedures in a detailed manner. These codes were used to evaluate the extent to which the four underlying models captured students' problem solving processes. The codes were also used to characterize processing differences between good and not-so-good problem solvers. In summary, the goals were:

- To develop a coding system for describing problem solving processes

- To test the adequacy of four models for describing problem solving processes

- To use the coding system to examine differences between good and not-so-good problem solvers.

The central method for addressing the questions in this study was the collection and analysis of verbal protocol ("think-aloud") data. Verbal protocols are open-ended think- 
aloud reports, through which participants are asked to verbalize what they are thinking as they work through a task.

\section{Four Problem Solving Models}

During the past several decades, extensive efforts have been directed at developing an ideal problem solving model. ${ }^{3}$ These efforts express the complexity of problem solving by incorporating cognitive, metacognitive, and attitudinal elements into problem-solving models. The models are expressed in specific terms, with the goal of making the processes of problem solving explicit, and thereby allowing educators to reflect on and incorporate the detailed processes of the models into effective instructional practices. The four models presented here are fleshed out in a manner that strives to present their elements in a uniform terminology and at a comparable level of expression. Formulating this level of descriptive consistency across the four models was a necessary step in developing a coding table that would allow a consideration of the adequacy of the models and meaningful comparisons of the models to students' problem solving behaviors, which reflect the goals of this study summarized above. The problem solving processes for each model were derived from a combination of authors' descriptions of problem solving in the text of their articles, as well as from the tables and figures in the respective papers.

The Wankat and Oreovicz Model (W). In considering problem solving processes, Wankat and Oreovicz ${ }^{1}$ note that novices tend to be anxious, have information organized into small pieces, do not know what information is relevant in the problem, reason from superficial problem details, jump to conclusions about what the problem is asking, do not analyze the problem into parts, often do not sketch the problem, use a trial and error strategy, do not check their solutions, and ignore corrective feedback. Experts, on the other hand, are typcially confident, organize information into "chunks," know what information is relevant in the problem, reason from fundamental principles, take time to define and redefine the problem to themselves, analyze the problem into parts, look for familiar patterns in the problem, spend considerable time sketching the problem, apply well-developed strategies, check their solutions, and learn from errors. The essential elements of problem solving in this model are summarized in Table 1a.

Table 1a. Problem-Solving Processes Based on the Wankat \& Oreovicz (1993, pp. 7172) Problem Solving Model (A Prestep and Six Operational Steps)

\begin{tabular}{ll}
\hline I Can & $1-$ Expresses anxiety or uncertainty \\
& $2-$ Expresses confidence \\
Define & - Lists knowns and unknowns \\
& 3 - Draws figure \\
& $4-$ Identifies constraints on the solution \\
& $1-$ Identifies criteria for solution \\
& $2-$ - Determines if there are required data that are unavailable in \\
Explore & the problem \\
& $3-$ Notes if entire problem is routine \\
& $4-$ Breaks the problem into parts
\end{tabular}


Plan

Do It

Check

Generalize

Other Processes
5 - Identifies parts/segments that are routine

6 - Considers alternative solution methods

7 - Considers the most convenient basis of representation

8 - Considers whether there is a more important underlying problem

9 - Calculates limits on the solution

1 - Develops logical structure of how to solve the problem

2 - Sets up steps to solve the problem

3 - Works through equations without numbers

1 - Inserts values into equations and calculates

1 - Checks calculations

2 - Compares answer to problem requirements in Define and

Explore

3 - Compares answer to common sense ("doesn't look right")

1 - Indicates what has been learned

2 - Indicates how they should have solved the problem to eliminate errors

3 -Considers how to solve the problem more efficiently in the future

Paraphrases and looks at different ways to interpret the problem Employs deep processing

Generalizes the problem, in order to understand it

Substitutes in numbers, in order to understand the problem

Simplifies problem (especially if stuck)

Relates the current problem to one he knows how to solve

(especially if stuck)

Searches for patterns (interconnected knowledge) instead of

single facts or elements

Changes the way the problem is being represented (especially if reaches obstacle in solution)

Retrieves memorized equations

Uses fundamental relations to generate equations

Considers whether solution plan is reasonable

Guesses the solution and then checks the answer

Monitors solution progress

If stuck, uses heuristics, perseveres, brainstorms

If stuck, guesses, quits

Uses broad experience to evaluate results

The Gray, Costanzo, \& Plesha Model (G). Gray et al. ${ }^{4}$ present a structured approach to problem solving. They regard this approach as a useful one to students throughout their careers. They developed this approach in response to students' use of a "hodgepodge of tricks" to solve statics and dynamics problems, but regard the method as able to guide students to the solution of any problem they encounter in mechanics. They present their method as "universally applicable" and appropriate for students as early as sophomorelevel mechanics. 
Gray et al. note that in solving homework and exam problems, students engage in "pattern matching" of the problem to equations they know "coming up with any $\mathrm{n}$ equations in $\underline{n}$ unknowns." In their structured approach they provide a set of basic equations from which students can derive the equations they need for a specific problem. The essential elements of problem solving in this model are summarized in Table 1b.

Table 1b. Problem-Solving Processes Based on the Gray et al. (2005) Structured Approach (from "Our Five Steps of Problem Solving”)

\begin{tabular}{ll}
\hline Road Map & 1 - Identifies given information \\
& 2 - Determines what needs to be solved for \\
& 3 - Outlines an overall solution strategy \\
& $1-$ Notes assumptions or idealizations in problem \\
Modeling & $2-$ Constructs free body diagram (model of problem) \\
& $1-$ Writes equations for solution \\
Governing & 2 - Organizes equations using key relations (equations) \\
Equations & 3 - Checks number of unknowns against number of equations \\
Computation & $1-$ Manipulates and solves equations \\
Discuss \& Verify & $1-$ Verifies that solution is correct \\
& $2-$ Considers what the solution physically means \\
& $3-$ Considers roles of assumptions in solution \\
& Chooses a coordinate system (e.g., Cartesian) \\
Other Processes & Assesses the adequacy of the problem model \\
& Assesses the accuracy of the problem model \\
& Checks signs on equations \\
& Compares reasonableness of solution (common sense) \\
\hline
\end{tabular}

The Litzinger, Van Meter, Wright, \& Kulikowich Model (L). Litzenger et al. ${ }^{5}$ were interested in problem analysis as a critical element in problem solving. Based on a review of the literature, they identified three factors that were closely related to analytical skills: content knowledge in the domain of the problem, knowledge of and ability to implement problem solving processes, and the ability to translate between representational systems, particularly translating between a verbal problem description and a diagrammatic depiction of the problem, like a free-body diagram. In their Integrated Problem Solving Model, these factors are separated into distinct dimensions of problem solving. To effectively solve a problem, a person must be able to integrate the processes of these dimensions. The essential elements of problem solving in this model are summarized in Table 1c.

Table 1c. Problem Solving Processes Based on Litzinger et al. (2006) Integrated Problem Solving Model

$\begin{array}{cl}\text { Problem } & 1 \text { - Reads problem } \\ \text { Representation } & 2 \text { - Activates prior knowledge } \\ \text { (Verbal) } & 3 \text { - Constructs a mental model } \\ & 4 \text { - Determines principles involved } \\ & 5 \text { - Determines deep structure of the problem }\end{array}$


6 - Recognizes familiar patterns

7 - Maps given information onto problem representation

8 - Determines what needs to be solved for

9 - Sets goals

10 - Sets subgoals

11 - Creates plans to achieve goals and subgoals

Problem 1 - Draws a diagram to represent the problem

Framing 2-Maps given information onto the problem representation

(Diagrammatic) 3-Maps prior knowledge onto problem representation

4 - Evaluates adequacy of the diagram

5 - Evaluates accuracy of the diagram

6 - Monitors problem framing process

7 - Detects errors and takes corrective action

Problem 1 - Calculates

Synthesis 2 -Monitors problem solving process

(Mathematical) 3-Evaluates solution

The Mettes, Pilot, Roossink, \& Kramers-Pals Model (M). Mettes et al. ${ }^{6}$ describe a systematic approach to problem solving instruction. Using a chart format, they present the flow of problem solving steps within their Systematic Approach to Solving Problems. From a pedagogical perspective, the authors adopt a constructivist approach to learning: that is, they emphasize that students must do their own learning and that instructors can only facilitate that learning. In constructivist terms, learning is an active process through which the learner constructs his or her own meaning; meaning is not simply transmitted from teacher to student. The essential elements of problem solving in this model are summarized in Table 1d.

Table 1d. Problem Solving Processes Based on Mettes et al. (1981) Systematic Approach to Problem Solving (SAP-Chart, p. 52)

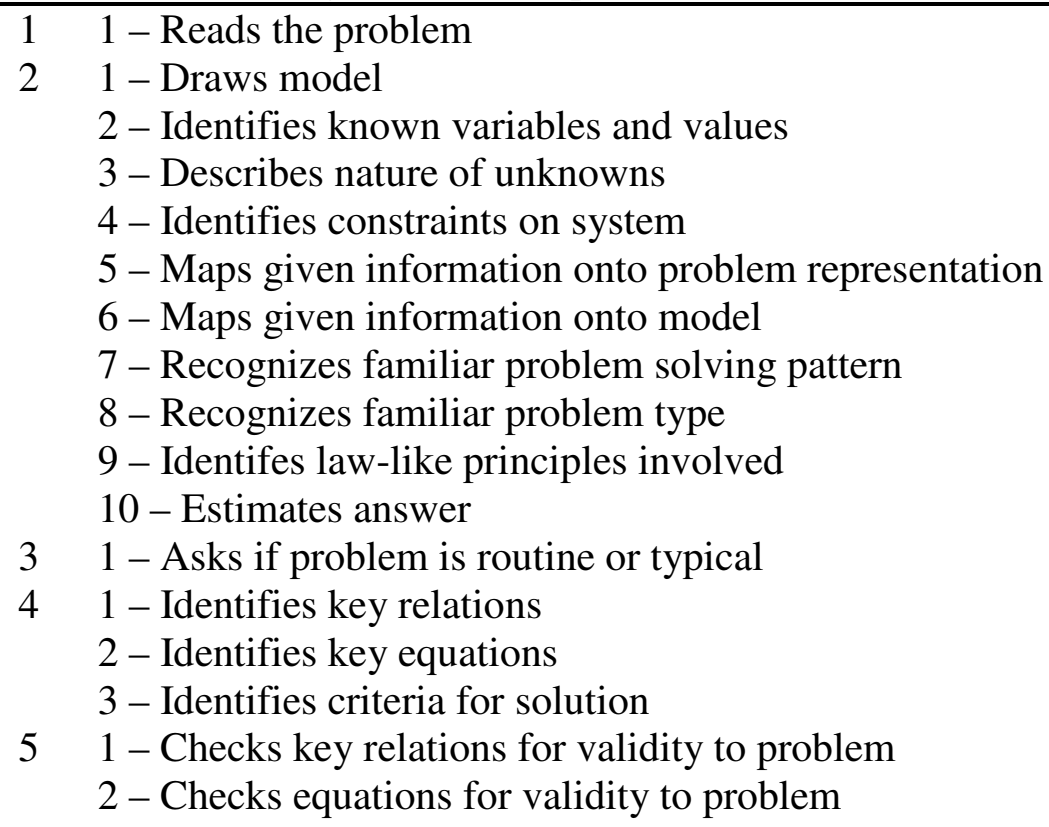


61 - Uses fundamental relations to generate equations

2 - Works through equations without numbers

3 - Sets up equation with specific values

$7 \quad 1$ - If not soluble, checks for missing key relations

2 - If not soluble, tries alternate problem-solving procedures

81 - Calculates answers

91 - Checks answer with sign, magnitude, or dimension

102 - Checks for mistakes in model

1 - Checks for mistakes on estimation

3 - Checks for mistakes on key relations

4-Checks for mistakes on calculations

The problem solving processes represented in the four models were incorporated into a comprehensive coding table, as described in the next section.

\section{Case Study}

The experimental methodology for addressing the questions in this study involved the collection and analysis of verbal protocol ("think-aloud") data. Verbal protocols are open-ended think-aloud reports, through which participants are asked to verbalize what they are thinking as they work through a task. Participants are instructed not to interpret or summarize the materials for the experimenter, unless those interpretations or summaries are a natural part of their thought processes. There are established precedents for using students' overt verbalizations to identify the cognitive representations that they construct while completing a task. ${ }^{7}$

A. Participants, Materials, and Procedure. Eighteen undergraduate students who were currently enrolled in Mechanics I and five faculty members were recruited through the engineering college at a large public university by one of the experimenters. The undergraduates were paid $\$ 25$ each for approximately one hour of participation. The five faculty members were from the Mechanical Engineering department. Additionally, two Mechanical Engineering faculty who did not provide verbal protocols graded participants' problem solutions, using a rubric provided by one of the experimenters (EEA). We are currently analyzing the complete corpus of verbal protocol data. For the present preliminary report, we randomly chose two faculty members, two higher-scoring students, and two lower-scoring students. The classification of students was based on the grades the students were assigned on the problems that they solved in this study. On a 100-point scale, where 100 is a perfect score, the average of the lower-scoring students was 57.25 points, and the average of the higher-scoring students was 87.50 points. The solutions of the two participating faculty members were also graded using the same grading rubrics and produced an average of 95.75 points.

Two problems were chosen that appeared in the exercises for Chapter 4 in Vector Mechanics for Engineers: Statics and Dynamics, $6^{\text {th }}$ Edition. $^{8}$ At the time of data collection, students had recently completed and had been tested in class on the material from that chapter. They had not, however, solved the two problems used in this study. 
The first problem statement read: The 10-m beam $\mathrm{AB}$ rests upon, but is not attached to, supports at $\mathrm{C}$ and $\mathrm{D}$. Neglecting the weight of the beam, determine the range of values of $\mathrm{P}$ for which the beam will remain in equilibrium. The second problem read: Neglecting friction, determine the tension in cable $\mathrm{ABD}$ and the reaction at support $\mathrm{C}$. Both problems were presented with the corresponding diagram from the textbook, and were assigned to participants in the same order as presented here.

Participants met individually with an experimenter in a quiet room. They were given instructions for the verbal protocol ("think-aloud") task. Then participants completed the task (solve two problems), which took approximately 50 minutes. The data were videorecorded for later transcription, with the permission of participants. During data collection, the primary role of the experimenter was to prompt participants to continue to verbalize their thoughts if they fell silent for an extended period. The verbatim instructions were as follows, with the textbook opened to the problems at the end of the chapter:

In this study, you will be asked to solve two statics problems from this chapter in your textbook. Take a moment to page through the chapter to confirm that you have covered this material. Each problem will be presented on a sheet of paper. Extra paper is available if you need it. Solve the problem as you normally would. But try to neatly show your work. As you are solving these problems, say out loud what you are thinking. The more thoughts you verbalize, the better. Whatever you say should simply reflect what is going through your mind while solving the problem. If you fall silent for more than a minute or so, I will remind you to keep talking. The textbook is available for you to use, you can use your personal notes, and you should feel free to ask questions at any time.

The verbal protocols were transcribed from the video-recordings. A preliminary coding table was constructed by compiling the problem solving processes from the four models described above. The processes were re-organized under the subheadings in Table 2 (e.g. Reading, Prior Knowledge, Knowns and Unknowns) in order to capture and reflect the conceptual coherence of subsets of the problem solving processes. In Table 2, the rows list the problem solving processes. The columns on the far right indicate which processes were included in each of the four models described earlier in this paper. A check mark is used to indicate that the process was mentioned in a particular model. Scanning across the four rows, process by process, for the four models readily shows that there was some consistency across models in emphasizing specific problem solving processes. However, it is also evident that the models differed considerably in describing essential problem solving processes. The rows in Table 2 for which there are no check marks reflect problem solving processes that were added into the coding table in the course of analyzing the verbal protocol data. These processes reflect elements of participants' behavior that were present in the data but that were not mentioned by any of the models.

Three of the experimenters conducted the verbal protocol analysis for the six participants in this study. This process involved coming to a unanimous agreement on two basic aspects of coding the data: parsing participants' utterances and assigning codes to the parsed segments. The convention adopted for parsing was to segment idea units, which 
were often indicated by noticeable pauses in a participant's speech pattern or a change in thought. The parsed segments were often clauses or sentences. The parsed segments also included the coding or reference to distinct elements of an equation or free-body diagram. If a parsed element could not be coded readily using available codes, then a new code was created. Coding was done in conjunction with observing the videorecording, which was focused on the participant's worksheet and textbook.

B. Results and Discussion. A total of 1696 utterances were parsed and coded for the six participants. The results of the analyses are summarized in Table 2. The data are reported as frequencies. That is, cells within Table 2 report the frequency with which the problem solving process listed for that row was mentioned by the participants. The data are presented for lower-scoring (LO) and higher-scoring (HI) participants, and for faculty (FA). The sum (SUM) is the total frequency of mention for the associated problem solving process.

Table 2. Coding Table Incorporating Problem-Solving Processes For Three Levels of Participants (LO: Lower-Scoring Undergraduates; HI: Higher-Scoring Undergraduates; FA: Faculty; SUM: the sum of the frequency with which a process was mentioned by the LO, HI, and FAC participants) and Four Models (W: Wankat \& Oreovicz, 1993; G: Gray et al., 2005; L: Litzinger et al., 2006; M: Mettes et al., 1981). Note: The numerical data in the table are the frequencies with which the associated problem solving processes were mentioned in the verbal protocol data.

\begin{tabular}{|c|c|c|c|c|c|c|c|c|c|}
\hline & & \multicolumn{3}{|c|}{ PARTICIPANTS } & & \multicolumn{4}{|c|}{ MODELS } \\
\hline & & $\mathrm{LO}$ & $\mathrm{HI}$ & FA & SUM & $\mathrm{W}$ & $\mathrm{G}$ & $\mathrm{L}$ & $\bar{M}$ \\
\hline 0 & Ambiguous or vague comment & 21 & & 9 & 30 & & & & \\
\hline 1 & Motivation & & & & & & & & \\
\hline $1 \mathrm{a}$ & Expresses anxiety or uncertainty & 1 & 3 & 1 & 5 & $\checkmark$ & & & \\
\hline $1 \mathrm{~b}$ & Expresses confidence & 1 & 2 & 2 & 5 & $\checkmark$ & & & \\
\hline & & & & & & & & & \\
\hline 2 & Reading & & & & & & & & \\
\hline $2 \mathrm{a}$ & Reads problem verbatim & 4 & 2 & 5 & 11 & & & $\checkmark$ & $\checkmark$ \\
\hline $2 \mathrm{~b}$ & Comments about problem & & 2 & 1 & 3 & & & & \\
\hline $2 \mathrm{c}$ & Re-reads problem & 18 & 2 & 4 & 24 & & & & \\
\hline $2 d$ & Checks textbook & 3 & & & 3 & & & & \\
\hline $2 \mathrm{e}$ & Checks textbook for analogous problem & 30 & & & 30 & & & & \\
\hline $2 \mathrm{f}$ & Checks textbook for support information & 8 & & & 8 & & & & \\
\hline 3 & Prior Knowledge & & & & & & & & \\
\hline $3 a$ & Activates prior knowledge & 8 & 3 & 13 & 24 & & & $\checkmark$ & \\
\hline $3 b$ & $\begin{array}{l}\text { Maps prior knowledge onto diagrammatic } \\
\text { representation }\end{array}$ & 6 & 4 & 2 & 12 & & & $\checkmark$ & \\
\hline $3 c$ & Maps prior knowledge onto equation & 4 & 3 & 2 & 9 & & & & \\
\hline $3 \mathrm{~d}$ & Maps earlier knowledge onto equation & 13 & & 8 & 21 & & & & \\
\hline 4 & Knowns and Unknowns & & & & & & & & \\
\hline $4 \mathrm{a}$ & Lists knowns and unknowns & 3 & 2 & 3 & 8 & $\checkmark$ & $\checkmark$ & & $\checkmark$ \\
\hline $4 \mathrm{~b}$ & Figures out knowns or unknowns & 4 & 4 & 3 & 11 & $\checkmark$ & $\checkmark$ & & $\checkmark$ \\
\hline $4 \mathrm{c}$ & Describes nature of unknowns & 5 & 4 & 7 & 16 & & & & $\checkmark$ \\
\hline $4 d$ & Maps givens into equation & 52 & 12 & 27 & 91 & & & & \\
\hline
\end{tabular}




\begin{tabular}{|c|c|c|c|c|c|c|c|c|c|}
\hline 5 & Principles & & & & & & & & \\
\hline $5 \mathrm{a}$ & Identifies law-like principles involved & & & & & & & $\checkmark$ & $\checkmark$ \\
\hline 6 & Familiar natterns & & & & & & & & \\
\hline $6 a$ & $\begin{array}{l}\text { Recognizes familiar or routine problem } \\
\text { type }\end{array}$ & & & 2 & 2 & $\checkmark$ & & $\checkmark$ & $\checkmark$ \\
\hline $6 \mathrm{~b}$ & $\begin{array}{l}\text { Recognizes familiar or routine problem } \\
\text { solving pattern }\end{array}$ & & 1 & 3 & 4 & $\checkmark$ & & $\checkmark$ & $\checkmark$ \\
\hline 7 & Goals & & & & & & & & \\
\hline $7 \mathrm{a}$ & Sets general goal & 2 & 3 & 6 & 11 & & & $\checkmark$ & \\
\hline $7 \mathrm{~b}$ & Sets subgoal & 1 & & 2 & 3 & & & $\checkmark$ & \\
\hline $7 \mathrm{c}$ & Creates plans to achieve goals and subgoals & 1 & & & 1 & & & $\checkmark$ & \\
\hline & & & & & & & & & \\
\hline 8 & Figures & & & & & & & & \\
\hline $8 \mathrm{a}$ & Selects coordinate system & & & 1 & 1 & & $\checkmark$ & & \\
\hline $8 \mathrm{~b}$ & Selects basis of representation & 1 & 2 & 3 & 6 & $\checkmark$ & & & \\
\hline $8 \mathrm{c}$ & Draws model (free body diagram) & 14 & 6 & 10 & 30 & $\checkmark$ & $\checkmark$ & $\checkmark$ & $\checkmark$ \\
\hline $8 \mathrm{~d}$ & Sketches part of problem & 5 & 1 & 5 & 11 & & & & $\checkmark$ \\
\hline $8 \mathrm{e}$ & $\begin{array}{l}\text { Maps given or known information onto } \\
\text { diagrammatic or system representation }\end{array}$ & 32 & 19 & 24 & 75 & & & $\checkmark$ & $\checkmark$ \\
\hline $8 \mathrm{f}$ & Evaluates adequacy of diagram (model) & & & 1 & 1 & & $\checkmark$ & $\bar{v}$ & \\
\hline $8 \mathrm{~g}$ & Evaluates accuracy of diagram (model) & 3 & 2 & & 5 & & $\checkmark$ & $\checkmark$ & \\
\hline $8 \mathrm{~h}$ & $\begin{array}{l}\text { Uses figure/diagrammatic representation to } \\
\text { reason about problem }\end{array}$ & 84 & 32 & 38 & 154 & & & & \\
\hline $8 \mathrm{i}$ & $\begin{array}{l}\text { Assigns new variable or value to } \\
\text { diagrammatic representation }\end{array}$ & 8 & 11 & 11 & 30 & & & & \\
\hline & & & & & & & & & \\
\hline 9 & Solution Development & & & & & & & & \\
\hline $9 \mathrm{a}$ & Proposes general explorative questions & 2 & 1 & 3 & 6 & $\checkmark$ & & & \\
\hline $9 \mathrm{~b}$ & $\begin{array}{l}\text { Considers alternative solution methods or } \\
\text { equations }\end{array}$ & 7 & 15 & 1 & 23 & $\checkmark$ & & & \\
\hline $9 \mathrm{c}$ & Identifies assumptions & 2 & 1 & 2 & 5 & & $\checkmark$ & & \\
\hline $9 \mathrm{~d}$ & $\begin{array}{l}\text { Maps given or known information onto } \\
\text { verbal representation }\end{array}$ & 19 & 1 & 11 & 31 & & & $\checkmark$ & \\
\hline $9 \mathrm{e}$ & Identifies constraints on system or solution & 7 & 24 & 28 & 59 & $\checkmark$ & & & $\checkmark$ \\
\hline 9f & Identifies criteria for solution & 3 & 1 & 2 & 6 & $\checkmark$ & & & $\checkmark$ \\
\hline $9 g$ & Determines what to solve for & 14 & 14 & 15 & 43 & & $\checkmark$ & $\checkmark$ & \\
\hline $9 \mathrm{~h}$ & $\begin{array}{l}\text { Considers or constructs deep structure, } \\
\text { mental model, or the underlying problem }\end{array}$ & 6 & 21 & 17 & 44 & $\checkmark$ & & $\checkmark$ & \\
\hline $9 \mathrm{i}$ & $\begin{array}{l}\text { Develops logical structure of how to solve } \\
\text { the problem }\end{array}$ & 1 & 5 & 2 & 8 & $\checkmark$ & $\checkmark$ & & \\
\hline $9 \mathrm{j}$ & $\begin{array}{l}\text { Considers whether solution plan is } \\
\text { reasonable }\end{array}$ & & & 2 & 2 & $\checkmark$ & & & \\
\hline $9 \mathrm{k}$ & Breaks problem into parts & & & 4 & 4 & $\checkmark$ & & & \\
\hline 91 & Sets up steps to solve the problem & & 4 & 3 & 7 & $\checkmark$ & $\checkmark$ & & \\
\hline $9 \mathrm{~m}$ & Estimates answer & 1 & 2 & 2 & 5 & & & & $\checkmark$ \\
\hline $9 n$ & $\begin{array}{l}\text { Guess the solution and then check the } \\
\text { answer }\end{array}$ & & 1 & & 1 & $\checkmark$ & & & \\
\hline 90 & Reasons about solution & 23 & 15 & 8 & 46 & & & & \\
\hline $9 p$ & $\begin{array}{l}\text { Determines the next step to solve the } \\
\text { problem }\end{array}$ & 11 & 5 & 8 & 24 & & & & \\
\hline & & & & & & & & & \\
\hline
\end{tabular}




\begin{tabular}{|c|c|c|c|c|c|c|c|c|c|}
\hline 10 & Equations & & & & & & & & \\
\hline $10 \mathrm{a}$ & $\begin{array}{l}\text { Identifies key relations that apply to } \\
\text { problem }\end{array}$ & 3 & 2 & 1 & 6 & & & & $\checkmark$ \\
\hline $10 \mathrm{~b}$ & $\begin{array}{l}\text { Identifies key equations that apply to } \\
\text { problem }\end{array}$ & 3 & 2 & 3 & 8 & & & & $\checkmark$ \\
\hline $10 \mathrm{c}$ & Retrieves memorized equations & & & & & $\checkmark$ & & & \\
\hline $10 \mathrm{~d}$ & $\begin{array}{l}\text { Uses key relations to generate or organize } \\
\text { equations }\end{array}$ & 23 & 7 & 9 & 39 & $\checkmark$ & $\checkmark$ & & $\checkmark$ \\
\hline $10 \mathrm{e}$ & Works through equations without numbers & & & & & $\checkmark$ & & & $\checkmark$ \\
\hline $10 f$ & $\begin{array}{l}\text { Sets up equation with specific variables / } \\
\text { values }\end{array}$ & 48 & 19 & 26 & 93 & $\checkmark$ & $\checkmark$ & & $\checkmark$ \\
\hline $10 \mathrm{~g}$ & $\begin{array}{l}\text { Checks number of unknowns against } \\
\text { number of equations }\end{array}$ & 7 & 2 & 4 & 13 & & $\checkmark$ & & \\
\hline $10 \mathrm{~h}$ & $\begin{array}{l}\text { Checks key relations for validity to } \\
\text { problem }\end{array}$ & & & & & & & & $\bar{v}$ \\
\hline $10 \mathrm{i}$ & Checks equations for validity to problem & 1 & 1 & & 2 & & & & $\checkmark$ \\
\hline $10 \mathrm{j}$ & Discusses equation with unknown in it & 1 & 7 & 2 & 10 & & & & \\
\hline $10 \mathrm{k}$ & Manipulates equation with unknowns & 54 & 45 & 78 & 177 & & & & \\
\hline 101 & Uses equation to reason about problem & 6 & 4 & 1 & 11 & & & & \\
\hline 11 & Calculations & & & & & & & & \\
\hline $11 \mathrm{a}$ & Calculates & 27 & 19 & 29 & 75 & $\checkmark$ & $\checkmark$ & $\checkmark$ & $\checkmark$ \\
\hline $11 \mathrm{~b}$ & Calculates limits on solution & & 1 & & 1 & $\checkmark$ & & & \\
\hline $11 \mathrm{c}$ & If stuck, guesses, quits & & & & & $\checkmark$ & & & \\
\hline $11 \mathrm{~d}$ & $\begin{array}{l}\text { If stuck, uses heuristics, perseveres, } \\
\text { brainstorms }\end{array}$ & 2 & 9 & 1 & 12 & $\checkmark$ & & & \\
\hline $11 \mathrm{e}$ & States answer & 12 & 7 & 10 & 29 & & & & \\
\hline 12 & Monitoring & & & & & & & & \\
\hline $12 \mathrm{a}$ & Monitors problem framing process & & 9 & 4 & 13 & & & $\checkmark$ & \\
\hline $12 b$ & $\begin{array}{l}\text { Detects errors in problem representation } \\
\text { and takes corrective action }\end{array}$ & 20 & 2 & 10 & 32 & & & $\checkmark$ & \\
\hline $12 \mathrm{c}$ & Monitors problem solving process & 11 & 12 & 22 & 45 & $\checkmark$ & & $\checkmark$ & \\
\hline $12 \mathrm{~d}$ & $\begin{array}{l}\text { If not soluble, checks for missing key } \\
\text { relations }\end{array}$ & & & & & & & & $\checkmark$ \\
\hline $12 \mathrm{e}$ & $\begin{array}{l}\text { If not soluble, tries alternate problem- } \\
\text { solving procedures }\end{array}$ & & 4 & & 4 & & & & $\checkmark$ \\
\hline 13 & Checking & & & & & & & & \\
\hline $13 a$ & Checks calculations & 1 & 2 & 9 & 12 & $\checkmark$ & & & $\checkmark$ \\
\hline $13 \mathrm{~b}$ & $\begin{array}{l}\text { Checks answer with sign, magnitude, and } \\
\text { dimensions }\end{array}$ & 5 & & & 5 & & $\checkmark$ & & $\checkmark$ \\
\hline $13 \mathrm{c}$ & $\begin{array}{l}\text { Compares solution to problem } \\
\text { requirements }\end{array}$ & 3 & 2 & 1 & 6 & $\checkmark$ & $\checkmark$ & $\checkmark$ & \\
\hline $13 \mathrm{~d}$ & Compares answer to common sense & 1 & & 2 & 3 & $\checkmark$ & $\checkmark$ & & \\
\hline $13 \mathrm{e}$ & Checks for mistakes on estimation & & & & & & & & $\checkmark$ \\
\hline $13 \mathrm{f}$ & Checks for mistakes in model & 1 & 3 & & 4 & & & & $\checkmark$ \\
\hline $13 \mathrm{~g}$ & Checks for mistakes on key relations & 1 & & & 1 & & & & $\checkmark$ \\
\hline $13 \mathrm{~h}$ & Confirms part of solution & 1 & 3 & 3 & 7 & & & & \\
\hline 14 & Reflection & & & & & & & & \\
\hline $14 \mathrm{a}$ & Considers what solution physically means & & & & & & $\checkmark$ & & \\
\hline $14 \mathrm{~b}$ & Considers role of assumptions in solution & & & & & & $\checkmark$ & & \\
\hline $14 \mathrm{c}$ & Makes observations on problem solving & & 3 & 2 & 5 & & & & \\
\hline
\end{tabular}




\begin{tabular}{|l|l|r|r|r|r|r|r|r|l|}
\hline & process after solution & & & & & & & & \\
\hline $14 \mathrm{~d}$ & Indicates what has been learned & & & & & $\checkmark$ & & & \\
\hline $14 \mathrm{e}$ & $\begin{array}{l}\text { Indicates how problem should have been } \\
\text { solved to eliminate errors }\end{array}$ & & & & & $\checkmark$ & & & \\
\hline $14 \mathrm{f}$ & $\begin{array}{l}\text { Considers how to solve the problem more } \\
\text { efficiently in the future }\end{array}$ & & & 1 & 1 & $\checkmark$ & & & \\
\hline $14 \mathrm{~g}$ & Uses broad experience to evaluate results & & & & & $\checkmark$ & & & \\
\hline & \multicolumn{1}{|c}{ Other } & & & & & & & & \\
\hline 15 & & 9 & 17 & 32 & 58 & & & & \\
\hline $15 \mathrm{a}$ & Provides explanatory comment & 4 & 20 & 11 & 35 & & & & \\
\hline $15 \mathrm{~b}$ & Makes self-reflective comment & 1 & 1 & 3 & 5 & & & & \\
\hline $15 \mathrm{c}$ & Orients to figure & 5 & 6 & 14 & 25 & & & & \\
\hline $15 \mathrm{~d}$ & Repeats comment & 678 & 439 & 579 & 1696 & 518 & 353 & 468 & 487 \\
\hline & TOTALS &
\end{tabular}

The full set of processes in Table 2 (excluding "Ambiguous or vague comment") are presented in response to our first goal above, which was to develop a coding system for describing problem solving processes. While it is true that additional codes may need to be added as the remaining protocols in our database are analyzed, it is also the case that this coding table has remained relatively stable while coding the latter three participants.

The second goal of this study was to test the adequacy of four models for describing problem solving processes. As the totals indicated for the columns associated with each of the models shown, the models captured between $21 \%$ (total frequency $=353$ ) and $31 \%$ (total frequency $=518$ ) of the 1696 coded utterances. These data are in no sense intended as a criticism of the respective models. Those authors formulated and presented their models with specific purposes. For instance, Litzenger et al. were concerned with central cognitive processes associated with problem analysis and the construction of a free-body diagram. Mettes et al. focused on presenting the essential elements for good instructional practices. Our goal, on the other hand, was to develop a coding rubric that could provide an exhaustive account of participants' observed problem-solving behaviors. The analysis presented here suggests that a comprehensive descriptive account of student and faculty problem solving requires a descriptive language that is richer than any set of descriptors that can be extracted from representative problem-solving models.

The third goal of this study was to use the coding system to examine differences between good and not-so-good problem solvers. It would be premature to draw conclusions from this small sample of data. Behavioral patterns should come into clearer focus as the remaining protocols are analyzed. When the full set of protocols is analyzed, we will systematically explore the data for cognitive and behavioral patterns that distinguish lower-scoring students from higher-scoring students. However, even at this point differences are emerging between the participants. A few key differences are indicated with bold font in the SUM column in Table 2 (e.g., processes $2 \mathrm{c}, 2 \mathrm{e}, 4 \mathrm{~d}$, $8 \mathrm{~h}$, etc.). Compared to higher-scoring students and faculty, lower-scoring students more frequently re-read the problem and checked the textbook for analogous problems. They spent a great deal of effort reasoning from the free-body diagram, essentially attempting to find some direction for constructing equations that would solve the problem. They reasoned more about the solution, but ineffectively, typically asking themselves how to set up 
equations to solve the problem. They drew on key relations (physical principles), primarily 'that the sum of forces about a point sum to zero,' but had difficulty formulating the equations using this principle that would reach the correct solution. They were less likely to construct a mental model or deep representation of the problem, instead operating from the surface features of the problem. They were less likely to identify constraints on the problem solution, which would narrow their options and point towards the appropriate solution path. They also showed less evidence of monitoring the problem solving process. Compared to lower-scoring students, higher-scoring students and faculty more frequently identified constraints on the problem solution, and they constructed mental models of the problem. Compared to students in general, faculty more frequently manipulated equations that led to a solution; in contrast, lower-scoring students spent much effort setting up equations using given information. Compared to students, faculty frequently monitored the problem solving process as they carried out the solution steps. This monitoring typically allows for strategic control over problem solving, such as switching from a fruitless solution path.

These findings generally agree with Litzenger et al. ${ }^{5}$ who reported that their student participants generally lacked quality knowledge, they failed to recall prior knowledge, and they did not recognize principles that applied to the problems that they were solving. For Litzenger et al., effective translational processes across representational systems verbal to diagrammatic to mathematical - are central components of successful problem solving. Litzenger et al. concluded that students did not have difficulty in translating from the verbal to diagrammatic. We also found this to be the case. Litzenger et al. did not examine the translation from diagrammatic to mathematical in their study. In our data, we have found that translation process to be especially difficult and one that has distinguished lower-scoring from higher-scoring students and faculty. Lower-scoring students were able to map givens from the verbal representation onto a free-body diagram. However, they struggled and generally failed in translating from the diagrammatic representation to the equations they needed to solve the problems. Litzenger et al. attributed students' greatest difficulties and challenges to prior knowledge and quality knowledge. Certainly part of the task of effectively translating from a freebody diagram to equations depends on prior knowledge that is sufficiently organized, accurate, and accessible.

\section{Conclusions}

The present study is concerned with the cognitive processes that students and faculty apply to typical engineering problems. A verbal protocol (think-aloud) methodology was adopted in order to capture participants' thought processes as they worked through textbook problems. Problem solutions consisted of reading the problem statement, drawing a free-body diagram, and developing relevant equations with regular reference to the free-body diagram. Participants were separated into faculty members, lower-scoring students, and higher-scoring students. A coding table was developed that reflected the cognitive processes associated with solving the problems. The results of the application of the coding scheme to participants' data led to the tentative conclusion that it is feasible to develop a comprehensive descriptive language for characterizing problem solving 
processes. Further, existing problem solving models capture some, but not a majority of the problem solving behaviors typically exhibited by undergraduates and faculty. An important goal of this study was to apply the coding scheme to participants who varied in problem solving ability in order to gain insight into the processes that distinguish skilled from less-skilled practitioners. The subset of data show little evidence overall for deeper and more reflective problem solving behaviors - e.g., those listed in Table 2 under "Checking" and "Reflection." Further, at the lower end of performance (LO participants), the frequency data indicate higher frequencies for processes associated with given information and equation construction. Those data, in conjunction with the lower grades those students received on the problems (avg. 57.27 points out of 100) suggest that lower-skilled students may require additional practice in translating the problem statement into a free-body diagram and coordinating that information with the construction of the correct equations for finding a solution. Additional data from this study and others like it will help to clarify the sources of differences of students with different skill levels. More comprehensive knowledge of what students actually do in the course of problem solving, like the data that we are exploring here, could eventually give some direction to instructional interventions. The process of coding and analyzing data like these is challenging and time-consuming. However, we hope that studies like these can ultimately benefit students through enhanced learning and instructional practices.

\section{Bibliography}

[1] Wankat, P.C., and Oreovicz, F.S., Teaching Engineering, New York, NY: McGraw-Hill, 1993.

[2] Taraban, R., M.W. Hayes, E.E. Anderson, and M.P. Sharma, "Giving Students Time for the Academic Resources That Work," Journal of Engineering Education, Vol. 93, No. 3, 2004, pp. 205-210.

[3] Woods, D. R., “An Evidence-Based Strategy for Problem Solving," Journal of Engineering Education, Vol. 89, No. 3, 2000, pp. 443-459.

[4] Gray, G. L., Costanzo, F., \& Plesha, M. E., "Problem Solving in Statics and Dynamics: A Proposal for a Structured Approach. Proceedings of the American Society for Engineering Education Annual Conference \& Exposition, Portland, OR, 2005.

[5] Litzinger, T., P. Van Meter, M. Wright, and J. Kulikowich, "A Cognitive Study of Modeling During Problem Solving," Proceedings of the American Society for Engineering Education Annual Conference \& Exposition, Chicago, IL, 2006.

[6] Mettes, C. T. C. W., Pilot, A., Roossink, H. J., \& Kramers-Pals, H., "Teaching and Learning Problem Solving in Science. Journal of Chemical Education, Vol. 58, No. 1, 1981, pp. 51-55.

[7] Ericsson, K.A., and H.A. Simon, Protocol Analysis: Verbal Reports as Data, Cambridge, MA: MIT Press, 1984.

[8] Beer, F. P., and Johnson Jr., E. R., Vector Mechanics for Engineers: Statics and Dynamics $\left(6^{\text {th }}\right.$ Ed.), Boston, MA: WCB/McGraw-Hill, 1997. 